\title{
Real-life management of patients with breakthrough cancer pain caused by bone metastases in Spain
}

This article was published in the following Dove Press journal: Journal of Pain Research

\author{
Carlos Ferrer Albiach (iD) \\ Francisco Villegas Estévez ${ }^{2}$ \\ $M^{a}$ Dolores López Alarcón ${ }^{3}$ \\ María de Madariaga ${ }^{4}$ \\ Alfonso Carregal ${ }^{5}$ \\ Javier Arranz ${ }^{6}$ \\ José M Trinidad Martín-Arroyo ${ }^{7}$ \\ Antonio Javier Jiménez López ${ }^{8}$ \\ Almudena Sanz Yagüe ${ }^{8}$ \\ 'Radiation Oncology Department, \\ Consorcio Hospital Provincial de \\ Castellón, Castellón, Spain; ${ }^{2}$ Pain Unit, \\ Consorcio Hospital Provincial de \\ Castellón, Castellón, Spain; ${ }^{3}$ Pain Unit, \\ Hospital General Universitario de \\ Valencia, Valencia, Spain; ${ }^{4}$ Pain Unit, \\ Hospital Universitario Infanta Sofía, \\ Madrid, Spain; ${ }^{5}$ Pain Unit, Complexo \\ Hospitalario Universitario de Vigo \\ (CHUVI), Pontevedra, Spain; ${ }^{6}$ Pain Unit, \\ Hospital Universitario Nuestra Señora de \\ Candelaria, Tenerife, Spain; ${ }^{7}$ Pain Unit, \\ Hospital Puerta del Mar, Cádiz, Spain; \\ ${ }^{8}$ Medical Department, Kyowa Kirin \\ Farmacéutica, S.L.U, Madrid, Spain
}

Correspondence: Carlos Ferrer Albiach Consorcio Hospital Provincial de Castellón, Avda Dr Clara 17, Castellón 12002, Spain

Tel +3 46I 8I7305I

Email carlos.ferrer@hospitalprovincial.es
Purpose: We aimed to explore the characteristics, and real-life therapeutic management of patients with breakthrough cancer pain (BTcP) caused by bone metastases in Spain, and to evaluate physicians' opinion of and satisfaction with prescribed BTcP therapy.

Participants and methods: For the purposes of this study, an ad-hoc questionnaire was developed consisting of two domains: a) organizational aspects and care standards; b) clinical and treatment variables of bone metastatic BTcP patients. In addition, physicians' satisfaction with their prescribed BTcP therapy was assessed. Specialists collected data from up to five patients receiving treatment for BTcP caused by bone metastasis, all patients gave their consent to participate prior to inclusion.

Results: A total of 103 cancer pain specialists (radiation oncologists [38.8\%], pain specialists [33.0\%], and palliative care (PC) specialists [21.4\%]) were polled, and data on 386 BTcP patients with bone metastatic disease were collected. Only $33 \%$ of the specialists had implemented specific protocols for BTcP management, and 19.4\% had established referral protocols for this group of patients. Half of all participants $(50.5 \%)$ address quality of life and quality of care in their patients; however, only $27.0 \%$ did so from the patient's perspective, as they should do. Most patients had multiple metastases and were prescribed rapid-onset fentanyl preparations $(71.2 \%)$, followed by immediate-release morphine $(9.3 \%)$ for the treatment of BTcP. Rapid-onset fentanyl was prescribed more often in PC units $(79.0 \%)$ than in pain units $(75.9 \%)$ and radiation oncology units $(61.1 \%) \quad(p<0.01)$. Furthermore, most physicians $(71.8 \%)$ were satisfied with the BTcP therapy prescribed.

Conclusions: Our results demonstrate the need for routine assessment of quality of life in patients with bone BTcP. These findings also underscore the necessity for a multidisciplinary therapeutic strategy for breakthrough pain in clinical practice in Spain.

Keywords: breakthrough cancer pain, bone metastases, management, health-related quality of life, opioids, satisfaction

\section{Introduction}

Pain is a major complication in patients suffering from advanced cancer. While the prevalence of pain in early-stage cancer is estimated at $33 \%$, this percentage nearly doubles in patients with metastatic disease. ${ }^{1,2}$

Cancer pain is classified into two distinct categories: persistent background pain, and transitory severe pain exacerbation, known as breakthrough cancer pain (BTcP). ${ }^{3}$

$\mathrm{BTcP}$ is a specific pain syndrome mainly caused by the neoplasm itself (70-80\% of cases). ${ }^{4}$ Breakthrough pain is often caused by bone metastases, which are common in patients with breast, prostate, and lung cancer. ${ }^{5,6}$ Most patients with bone metastases 
experience acute severe pain that is often localized to a particular area. ${ }^{7,8}$ As cancer pain progresses in severity it can aggravate the physical impairment and movement limitation produced by bone metastasis, and can also severely undermine the patient's health-related quality of life (HRQoL). ${ }^{5,9}$ Pain related to bone metastasis should be managed with analgesic drugs. According to the World Health Organization (WHO), mild pain should be treated with NSAIDs (non-steroidal antiinflammatory drugs) and paracetamol, whereas weak opioids are the treatment of choice for mild to moderate pain. Finally, moderate to severe pain should be managed with strong opioids. ${ }^{10-12}$ In this context, the onset of intense breakthrough pain demands fast-acting therapy to control the pain and improve functionality in bone metastatic patients. There are numerous pharmaceutical and non-pharmaceutical options currently available for BTcP, a condition that presents a significant challenge to medical specialists.

In this context, rapid-onset opioids (ROOs) have been shown to provide faster acting and more effective pain relief than traditional immediate-release morphine or placebo. ${ }^{13}$ Based on this evidence, the latest versions of the Spanish Society of Medical Oncology (SEOM) ${ }^{4}$ and the European Society for Medical Oncology (ESMO) ${ }^{14}$ guidelines consider ROOs to be more suited to breakthrough pain than immediate-release morphine, and recommend them as the first option to treat BTcP.

Despite these recommendations and the growing evidence supporting the use of ROOs in recent years, some studies ${ }^{15,16}$ suggest that a high proportion of cancer patients receive suboptimal analgesia. Undertreatment of bone pain is also common, adding a further burden to metastatic disease. ${ }^{17}$ These data show the need to improve the treatment strategy in patients with bone-related BTcP. However, the management of BTcP in metastatic patients in clinical practice is poorly characterized in our setting. Providing such real-world evidence would help evaluate improvements in BTcP management in future studies. For this purpose, we aimed to explore the characteristics and real-life therapeutic management of patients with BTcP caused by bone metastases. Additionally, we sought to evaluate physician satisfaction with the prescribed BTcP therapy, to ascertain their opinion regarding the key aspects of $\mathrm{BTcP}$ treatment, and to identify points of agreement and discrepancy among different specialties involved in treating bone metastatic cancer.

\section{Materials and methods}

An electronic questionnaire was specifically designed to collect real-world data on the management of BTcP associated with bone metastases. It was divided into two domains: 1) organizational aspects and BTcP care standards according to physician opinion; 2) clinical and treatment variables of bone were also assessed. In the second domain, specialists collected clinical data from their patients. For inclusion, patients were required to be older than 18 years, to have received a diagnosis of $\mathrm{BTcP}$ caused by bone metastases, and to be receiving treatment for BTcP.

The following organizational aspects of medical units were collected in domain 1) doctors and nurses working full time, catchment area, number of patients with BTcP and metastatic disease seen last month, referral protocol for patients with cancer pain and BTcP associated with metastatic disease, treatment guidelines for cancer pain and $\mathrm{BTcP}$ associated with metastatic disease, patient information fact sheets about treatment, regular multidisciplinary tumor board meetings, use of quality of life questionnaires. Physicians were also asked about the percentage of patients with bone BTcP who should be treated with different therapeutic options, which treatment they recommended for BTcP (Likert scale 1-7: 1 never recommended; 7 always recommended), which were the major determinants for the choice of the BTcP treatment (Likert scale 1-7: 1: never taken into account; 7: always taken into account), their level of satisfaction with BTcP treatment in general (Likert scale: $1=$ extremely dissatisfied; $7=$ extremely satisfied) and the major determinant of BTcP treatment efficacy (placed in order, 1 being the most important and 5 the least important).

The clinical variables of bone metastatic BTcP patients evaluated in domain 2) were: location of the primary tumor, number of metastases, patient performance status (ECOG scale and Karnofsky Index), characteristics of baseline pain (type and intensity on a visual analog scale [VAS]), pharmacological treatment for baseline pain, characteristics of BTcP episodes during the week prior to data collection (number of $\mathrm{BTcP}$ episodes per day, duration of BTcP episodes, pain intensity [VAS]; BTcP occurrence and cause), pharmacological treatment for BTcP and physicians' satisfaction with this treatment ( $1=$ extremely dissatisfied; $7=$ extremely satisfied). We also recorded if QoL had been determined.

Participants were pain management specialists mainly from radiation oncology $(\mathrm{RO})$ units, palliative care (PC) units, and pain units (PU). All were required to have experience in diagnosing and treating cancer pain. Specialists collected data from up to five patients, and all patients gave their consent to participate. The study was approved by the Ethics Committee of the Consorcio Hospitalario Provincial de Castellón. 
Data were analyzed descriptively using $\mathrm{R}$ statistics 3.2.5. Categorical variables were described as absolute and relative frequencies; central tendency and dispersion were reported for quantitative variables. The Chi-square test was used for comparisons among different specialties. Statistical significance was set at $p<0.05$.

\section{Results}

A total of 103 cancer pain specialists from RO $(n=40 /$ $103 ; 38.8 \%)$, pain $(n=34 / 103 ; 33.0 \%)$, and $\mathrm{PC}(\mathrm{n}=22 / 103$; $21.4 \%)$ units were polled. Mean $( \pm \mathrm{SD})$ years of experience

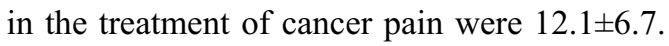

\section{Care standards in specialized units}

Answers to questions about care standards in specialized units are summarized in Table 1.

Most physicians polled ( $\mathrm{n}=97 / 103 ; 94.2 \%)$ were attached to units with a catchment area of $>100,000$ inhabitants. Care units had a mean of $4.9 \pm 3.4$ full-time physicians and 3.8 \pm 3.6 full-time nurses. Most respondents $(\mathrm{n}=77 / 103 ; 74.7 \%)$ estimated that between 5 and 25 BTcP patients with bone metastases had been seen in their units in the preceding month.

According to pain experts, most of their hospitals had medical oncology departments $(\mathrm{n}=96 / 103 ; 93.2 \%)$, PU $(\mathrm{n}=90 / 103 ; 87.4 \%)$, or PC units $(\mathrm{n}=87 / 103 ; 84.5 \%)$; however, only $19.4 \%(n=20 / 103)$ had implemented a referral protocol for monitoring patients with $\mathrm{BTcP}$ and metastatic disease.

Most physicians $(>60 \%)$ reported that their units provide patients with printed fact sheets about pain treatment and had protocols for cancer pain treatment and assessment. However, only $33 \%(n=34 / 103)$ had established a specific protocol for BTcP management in patients with bone metastases. When participants were asked about the routine use of HRQoL questionnaires, around half $(\mathrm{n}=52$ / $103 ; 50.5 \%$ ) reported that they address quality of life or quality of care of their BTcP patients. However, only $27.0 \%(n=24 / 89)$ of specialists routinely evaluate quality of care from the patient's perspective in clinical practice.

\section{BTcP therapeutic management according to specialists}

According to the physicians polled, at least half of all patients suffering from bone $\mathrm{BTcP}$ should be treated with strong opioids ( $\mathrm{n}=98 / 103 ; 95.1 \%$ ), followed by analgesic radiotherapy $(\mathrm{n}=87 / 103 ; 84.4 \%)$ or coanalgesics $(\mathrm{n}=72 / 103 ; 69.9 \%)$, while fewer specialists considered that breakthrough pain due to bone metastases should be managed with interventional techniques $(n=37 / 103 ; 35.9 \%)$ or weak opioids $(n=10 /$ $103 ; 9.7 \%)$. In general, there were no significant differences between specialties, although most physicians that considered interventional techniques to be appropriate belonged to PUs ( $70.3 \%$ vs $21.4 \%$ for PC and $5.4 \%$ for RO).

Among pharmacotherapies, most respondents recommended transmucosal fentanyl as their treatment of choice for BTcP caused by bone metastases, followed by parenteral opioids $(1=$ least recommended and $7=$ most recommended $)$ (Figure 1). There were significant differences between specialties, with more PC physicians choosing parenteral opioids (PC: $5.6 \pm 1.4$ vs PU: $3.6 \pm 1.9$ and RO: $3.7 \pm 2.0$; $p<0.0001)$. There were also significant differences regarding oral transmucosal fentanyl (PC: $6.7 \pm 0.8$ vs PU: $6.6 \pm 0.7$ vs RO: $6.2 \pm 1.1 ; p<0.05)$ and nasal transmucosal fentanyl (PC: $6.6 \pm 0.9$ vs PU: $6.4 \pm 0.7$ vs RO: $5.9 \pm 1.4 ; p<0.05$ ) between the three care units. Moreover, experts rated their degree of satisfaction with BTcP therapy ( $1=$ extremely dissatisfied; $7=$ extremely satisfied) with a mean score of 5.4 \pm 0.9 , with the highest score reported in ROs: $5.8 \pm 0.7$ followed by PUs: $5.2 \pm 0.9$ and PCs: $5.1 \pm 1.0$.

When deciding on the most suitable treatment to address BTcP caused by bone metastases, most experts considered the onset of action $(\mathrm{n}=80 / 103 ; 77.7 \%)$ and duration of analgesia $(n=71 / 103 ; 68.9 \%)$ or the route of administration $(\mathrm{n}=68 / 103 ; 66.0 \%)$ and the titration method $(n=64 / 103 ; 62.1 \%)$ of the pharmacotherapy. Other characteristics, such as the patient's social support $(n=39 / 103$; $37.9 \%$ ), were less frequently considered when weighing up treatment options. According to most experts, the major determinant of BTcP treatment efficacy was pain intensity $(\mathrm{n}=53 / 103 ; 51.5 \%)$, followed by the frequency of BTcP episodes $(n=28 / 103 ; 27.2 \%)$, patient functionality $(n=16 /$ $103 ; 15.5 \%)$, presence of adverse events $(n=4 / 103 ; 3.9 \%)$ and last, the duration of BTcP episodes $(\mathrm{n}=2 / 103 ; 1.9 \%)$.

\section{Real-world data of patients with BTcP caused by bone metastases Characteristics of patients and BTcP episodes}

Physicians collected data from $386 \mathrm{BTcP}$ patients with metastatic bone disease, of whom $68.1 \%(n=263 / 386)$ were men; mean age was $65.7 \pm 12.2$ years. Primary tumors were mainly localized in the lungs ( $\mathrm{n}=98 / 386 ; 25.4 \%)$ and prostate $(\mathrm{n}=85 / 386 ; 22.0 \%)$. In $74.9 \%(\mathrm{n}=289 / 386)$ of cases, patients had multiple bone metastases. In addition, more than $70 \%(n=278 / 386)$ of patients had an ECOG 
Table I Organizational aspects of care units

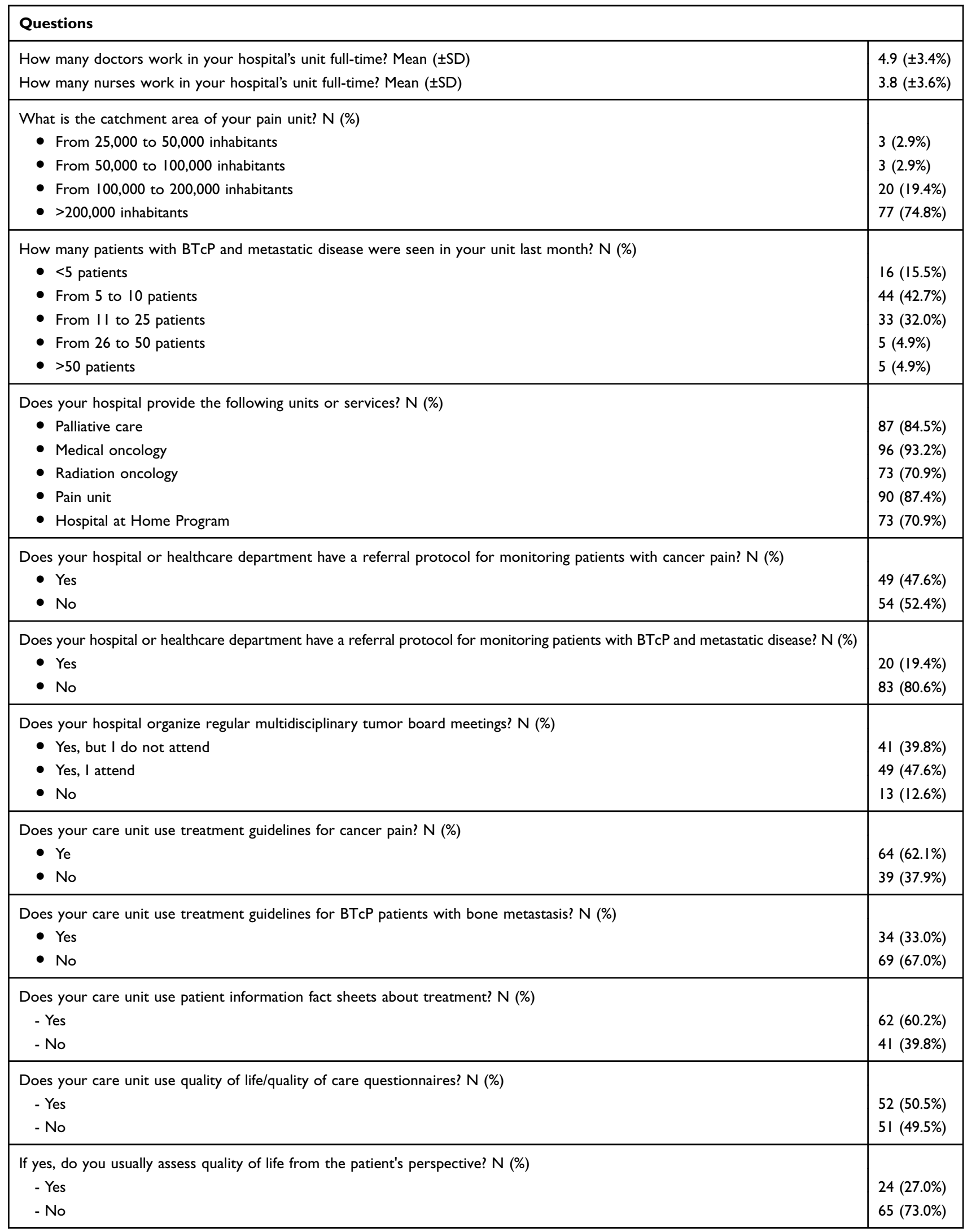

Abbreviations: BTcP, breakthrough cancer pain; SD, Standard Deviation. 


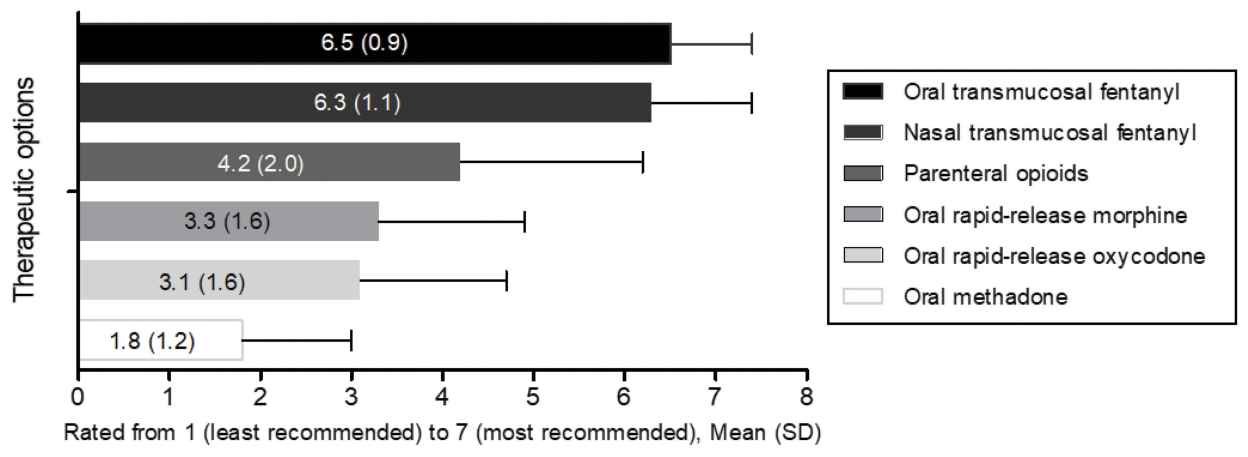

Figure I Physician grade of recommendation for BTcP therapeutic.

Notes: Physician grade of recommendation for BTcP therapeutic options was assessed using a 7-point Likert scale (where I= least recommended and 7= most recommended). Abbreviation: BTcP, breakthrough cancer pain.

performance status of 1 or $2(n=278 / 386 ; 72.1 \%)$ and a Karnofsky Performance score of between 50 and 80 $(\mathrm{n}=289 / 386 ; 74.9 \%)$. Patients had a mean of $3.5 \pm 1.8$ BTcP episodes per day, mainly characterized by sudden onset, severe intensity (on VAS) and a mean duration of $22.2 \pm 17.5$ mins (Table 2).

HRQOL had been assessed in $19.2 \%(n=74 / 386)$ of patients. The cancer-specific EORTC quality of life questionnaire C-30 (EORTC QLQ-C30) was the most commonly used questionnaire, followed by the generic 12-Item Short Form Health Survey (SF-12).

\section{Pharmacological treatment of BTcP associated with bone metastasis}

Baseline pain was mainly controlled with transdermal fentanyl $(n=160 / 386 ; 41.5 \%)$, oral morphine $(n=35 / 386$; $9.1 \%)$ or tapentadol rectally administered $(n=27 / 386$; 7.0\%) (Supplementary Table 1).

Regarding BTcP treatment, most patients $(n=275 / 386$; $71.2 \%$ ) were prescribed rapid-onset fentanyl preparations alone or in combination with other analgesics, followed by immediate-release morphine $(\mathrm{n}=36 / 386 ; 9.3 \%)$ and NSAIDs $(n=21 / 386 ; 5.4 \%)$ (Figure 2; Supplementary Tables 2 and 3). The most common route of fentanyl administration among BTcP patients was sublingual $(n=187 / 275 ; 68.0 \%)$, followed by nasal $(n=36 / 275 ; 13.1 \%)$ and oral $(n=21 / 275 ; 7.6 \%)$. The mean fentanyl dose was $214 \pm 171 \mu \mathrm{g}$. Fentanyl was used on demand, with a frequency of administration of one dose every $4-6 \mathrm{hrs}$ in $55.3 \%$ of patients. In most cases $(n=266 / 386$; $68.9 \%$ ), medication was titrated to the lowest effective dose. Dose adjustments had been made for baseline pain management and patient compliance had been assessed before BTcP treatment prescription in most cases. Additionally, fentanyl preparations alone were mainly prescribed when BTcP intensity was severe $(n=120 / 170,70.6 \%)$, and BTcP occurred suddenly $(n=108 / 170,63.5 \%)$ and unpredictably $(n=93 / 169$, $55.0 \%$ ). Approximately half of these cases (49.4\%) were spontaneous ( $\mathrm{n}=84 / 170)$ (Supplementary Table 4).

Rapid-onset fentanyl was the most common medication prescribed in all specialized units; however, PC physicians reported significantly higher rates of prescription compared to RO $(79.0 \%$ vs $61.1 \% ; p<0.01)$ and PU $(79.0 \%$ vs $75.9 \% ; p=0.056$ ). The second therapeutic option in PCs was immediate-release morphine, while NSAIDs were the second most common option in PUs (Figure 3). Furthermore, sublingual fentanyl was prescribed more frequently in PCs (81.4\%) than in ROs (79.1\%) and PUs $(69.5 \%)$, although these differences were not statistically significant $(p>0.05)$. In turn, $28 \%$ of the patients $(n=108)$ were treated with a combined strategy of pharmaceutical treatment and interventional techniques. The most commonly used techniques for BTcP treatment caused by bone metastases were peripheral nerve blockade (35.2\%) and epidural block $(25.0 \%)$.

Most physicians (71.8\%) were satisfied to some extent with the prescribed BTcP therapy, $15.0 \%(n=58 / 386)$ were neutral, and $13.2 \%(n=51 / 386)$ were dissatisfied with the analgesia given (Figure 4). Mean satisfaction with treatment was $5 \pm 1.4$ ( $1=$ extremely dissatisfied, $7=$ extremely satisfied). According to physicians, dissatisfaction with BTcP treatment most frequently resulted from departmental organization issues $(\mathrm{n}=114 / 439 ; 26.0 \%)$, while only $13.2 \%(n=58 / 439)$ cited lack of therapeutic efficacy as the main cause of dissatisfaction.

\section{Discussion}

This study provides a comprehensive description of the characteristics and therapeutic management of patients 
Table 2 Characteristics of BTcP patients and BTcP episodes

\begin{tabular}{|c|c|}
\hline \multicolumn{2}{|l|}{ Characteristics of BTcP patients } \\
\hline Age, years $( \pm S D)$ & $65.7(12.2)$ \\
\hline \multicolumn{2}{|l|}{ Gender, n (\%) } \\
\hline Male & $263(68.1)$ \\
\hline Female & $123(31.9)$ \\
\hline \multicolumn{2}{|l|}{ Location of the primary tumor, $\mathrm{n}(\%)$} \\
\hline Lung & $98(25.4)$ \\
\hline Prostate & $85(22.0)$ \\
\hline Gastrointestinal & $64(16.6)$ \\
\hline Breast & $63(16.3)$ \\
\hline Others & $76(19.7)$ \\
\hline \multicolumn{2}{|l|}{ Quantity of bone metastases, $n$ (\%) } \\
\hline Multiple & $289(74.9)$ \\
\hline Single & $95(24.6)$ \\
\hline NA & $2(0.5)$ \\
\hline \multicolumn{2}{|l|}{ ECOG score, n (\%) } \\
\hline ECOG 0 - Fully active, able to carry on all pre-disease performance without restriction. & $26(6.7)$ \\
\hline $\begin{array}{l}\text { ECOG I - Restricted in physically strenuous activity but ambulatory and able to carry out work of a light or sedentary nature, } \\
\text { e.g., light house work, office work }\end{array}$ & $106(27.5)$ \\
\hline ECOG 2 - Ambulatory and capable of all self-care but unable to carry out any work activities. Up and about more than $50 \%$ of waking hours & $172(44.6)$ \\
\hline ECOG 3 - Capable of only limited self-care, confined to bed or chair more than $50 \%$ of waking hours & $69(17.9)$ \\
\hline ECOG 4- Completely disabled. Cannot carry on any self-care. Totally confined to bed or chair & $12(3.1)$ \\
\hline \multicolumn{2}{|l|}{ Karnofsky Index, n (\%) } \\
\hline 20- Very sick; hospital admission necessary; active supportive treatment necessary & $4(1.0)$ \\
\hline 30- Severely disabled; hospital admission is indicated although death not imminent. & $8(2.1)$ \\
\hline 40- Disabled; requires special care and assistance. & $36(9.3)$ \\
\hline 50- Requires considerable assistance and frequent medical care. & $62(16.1)$ \\
\hline 60- Requires occasional assistance but is able to care for most of their personal needs. & $60(15.5)$ \\
\hline 70- Cares for self; unable to carry on normal activity or to do active work. & $97(25.1)$ \\
\hline 80- Normal activity with effort; some signs or symptoms of disease. & $70(18.1)$ \\
\hline 90- Able to carry on normal activity; minor signs or symptoms of disease. & $41(10.6)$ \\
\hline I00- Normal; no complaints; no evidence of disease. & $8(2.1)$ \\
\hline \multicolumn{2}{|l|}{ Characteristics of basal pain } \\
\hline \multicolumn{2}{|l|}{ Type, n (\%) } \\
\hline Mixed & $231(59.8)$ \\
\hline Neuropathic & $32(8.3)$ \\
\hline Nociceptive & $114(29.5)$ \\
\hline Procedural & $8(2.1)$ \\
\hline Unknown & $\mathrm{I}(0.3)$ \\
\hline Pain intensity (VAS), mean (SD) & $6.2(2.1)$ \\
\hline \multicolumn{2}{|l|}{ Characteristics of BTcP } \\
\hline Number of BTcP episodes per day, mean (SD) & $3.5(1.8)$ \\
\hline Duration of BTcP episodes, mean (SD) & $20.2(17.5)$ \\
\hline \multicolumn{2}{|l|}{ Pain intensity (VAS), n (\%) } \\
\hline Severe (VAS 7-10) & $275(7 I .2)$ \\
\hline Moderate (VAS 4-6) & $103(26.7)$ \\
\hline Mild (VAS 0-3) & $8(2.1)$ \\
\hline
\end{tabular}

(Continued) 
Table 2 (Continued)

\begin{tabular}{|l|l|}
\hline Characteristics of BTcP patients & \\
\hline $\begin{array}{l}\text { BTcP occurrence, } n(\%) \\
\text { Sudden onset } \\
\text { Progressive onset }\end{array}$ & $236(61.1)$ \\
\hline $\begin{array}{l}\text { BTcP occurrence, } n(\%) \\
\text { Unpredictable }\end{array}$ & $150(38.9)$ \\
Predictable & $214(55.4)$ \\
No answer & $171(44.3)$ \\
\hline $\begin{array}{l}\text { Cause of BTcP, } n(\%) \\
\text { Spontaneous pain } \\
\text { Incidental pain }\end{array}$ & $1(0.3)$ \\
\hline
\end{tabular}

Abbreviations: BTcP, breakthrough cancer pain; ECOG, Eastern Cooperative Oncology Group; VAS, visual analog scale.

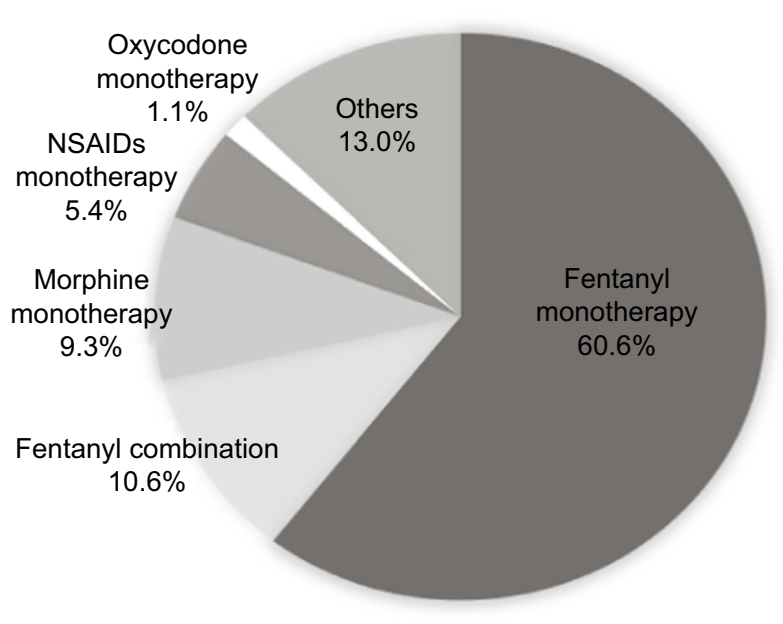

Figure 2 Distribution of patients according to type of BTcP treatment. Abbreviations: BTcP, breakthrough cancer pain; NSAIDS, nonsteroidal antiinflammatory drugs.

with BTcP caused by bone metastases in clinical practice. $\mathrm{BTcP}$ is a common manifestation of bone malignancy, and greatly affects the emotional and physical health of patients. However, very few studies have previously evaluated BTcP management in this population.

Successful management of BTcP can require a multidisciplinary approach that involves common standards of care and the establishment of referral protocols between services involved in patient management. ${ }^{18}$ According to the experts polled, very few departments used treatment protocols and specific referral criteria for BTcP patients with bone metastases. These findings underline the need for multidisciplinary strategies to manage BTcP in this specific group of patients.

BTcP management requires the assessment of psychosocial factors, such as social support, cognitive status, or psychological stress, all of which can affect therapeutic efficacy. ${ }^{19}$ Clinical guidelines agree that cancer pain management in incurable cancer is best provided as part of a multiprofessional PC approach, and all other domains of suffering (psychosocial, spiritual, and existential) need to be carefully addressed («total pain»). ${ }^{20}$ However, according to our survey only $37.9 \%$ of the physicians factored

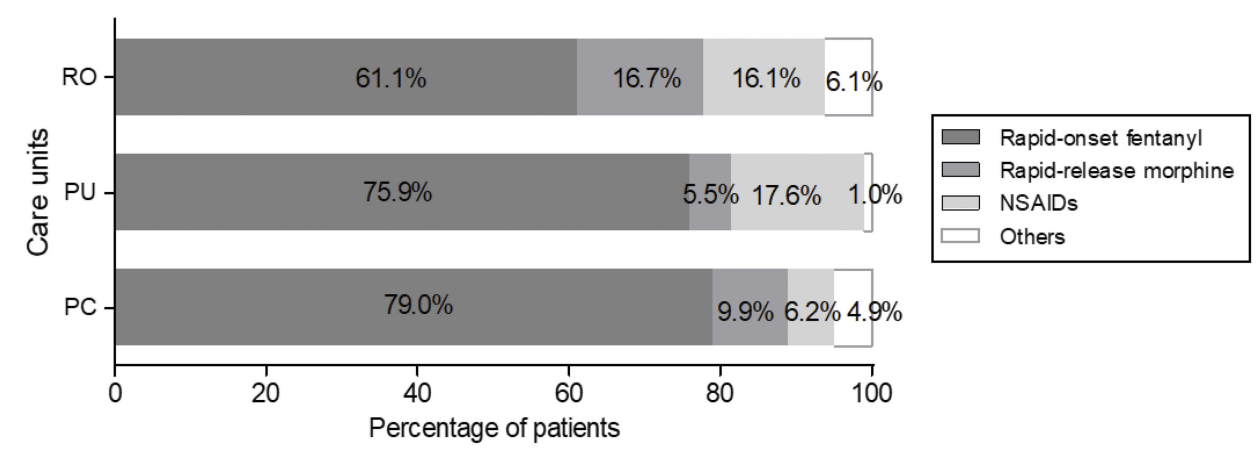

Figure 3 Distribution of patients according to type of BTcP treatment and care units.

Abbreviations: BTcP, breakthrough cancer pain; NSAIDS, nonsteroidal anti-inflammatory drugs; PC, palliative care; PU, pain units; RO, radiation oncology. 


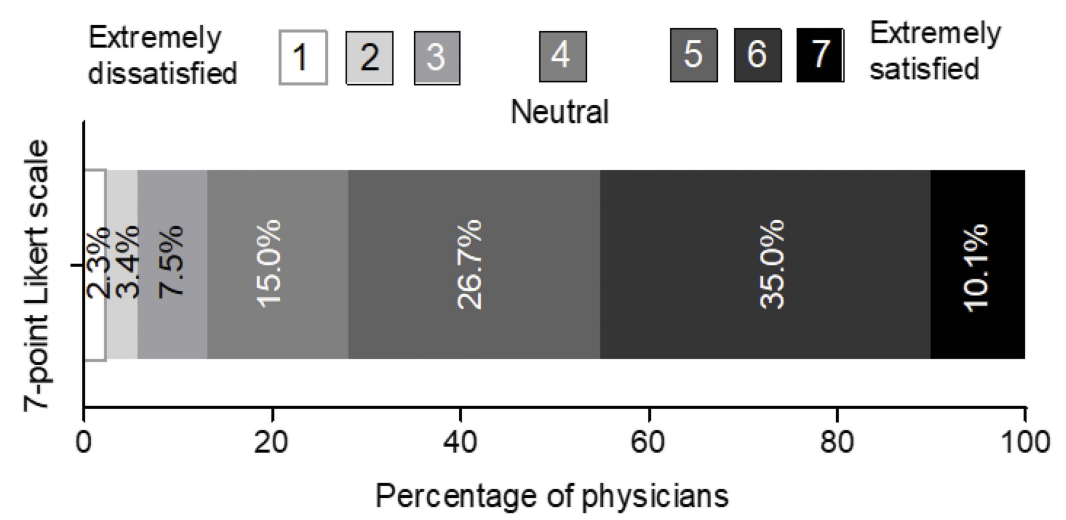

Figure 4 Distribution of physicians according to degree of satisfaction with BTcP therapy.

Notes: Physician satisfaction with treatment was assessed using a 7-point Likert scale (where I= extremely dissatisfied and $7=$ extremely satisfied).

Abbreviation: BTcP, breakthrough cancer pain.

social support into their bone metastases-induced BTcP treatment decision-making.

In addition, our results show that HRQoL assessment tools are not routinely used in patients with metastatic disease. This is consistent with findings reported in other studies, ${ }^{21}$ suggesting that physicians do not consider measuring quality of life as part of therapeutic management. Several studies have shown that BTcP affects a patient's emotional and physical health as well as their capacity to perform routine tasks and to participate in social activities. $^{21-23}$ Because of this, cancer patients consider HRQoL to be as important as survival. ${ }^{22}$

Quality of life assessment is also important in patients diagnosed with bone metastases. Although they are generally considered to have limited survival prospects, significant progress in anticancer drugs in the last years has increased their life expectancy. In addition, these patients often suffer skeletal-related events such as fracture which might reduce mobility and increase both pain and anxiety, ${ }^{24}$ but our clinical practice data confirmed that HRQoL was rarely included as a BTcP treatment outcome in metastatic patients.

Data from cancer patients with multiple bone metastases showed that they had several BTcP episodes per day. BTcP episodes were mainly characterized by sudden and unpredictable onset, severe intensity, and a mean duration of $22.2 \pm 17.5$ mins, which is somewhat longer than that reported in a broader cancer patient population. ${ }^{25}$ The ECOG performance status and Karnofsky index score of patients in our study showed that most have impaired functionality that limits their capacity to perform daily activities and to work full time. Despite this, only $15.5 \%$ of the specialists considered functionality to be the major determinant of BTcP treatment efficacy.
Conversely, most participants, regardless of their medical specialty, agreed that strong opioids, analgesic radiotherapy, and coanalgesics were the best options for treating BTcP in bone metastatic patients. Radiotherapy has traditionally been the gold-standard treatment for pain induced by bone metastases; however, studies have shown that this only achieves complete pain relief in a small percentage of patients, ${ }^{26}$ prompting some authors to suggest that bone metastatic pain requires both pharmacological and radiation therapies. ${ }^{27,28}$ In particular, current ESMO guidelines recommend a single 8 Gy dose of external beam radiotherapy in association with analgesics for patients with painful bone metastases (level of evidence I, degree of recommendation $\mathrm{A}){ }^{14}$

The use of interventional techniques in the treatment of $\mathrm{BTcP}$ has been controversial. Many guidelines recommend that these interventions should be considered for BTcP in accordance with patient needs. ${ }^{29-31}$ However, even though these therapies can prevent $\mathrm{BTcP}$ and may improve quality of life, their analgesic efficacy has never been properly assessed. $^{32}$

Regarding pharmacological treatment, the specialists polled recommended ROOs, and more specifically transmucosal fentanyl formulations, over immediate-release oral morphine or oxycodone. Immediate-release morphine has traditionally been the first-line rescue medication for BTcP, and is still considered the first therapeutic option by some clinical practice guidelines. ${ }^{29}$ However, following approval of transmucosal fentanyl, oral morphine has been relegated to some selected cases. Specifically, the ESMO guidelines ${ }^{14}$ recommend the use of ROOs as the first choice for the treatment of unpredictable and rapid-onset BTcP (level of evidence I, degree of recommendation A), and suggest limiting oral 
opioids such as morphine to the treatment of predictable pain (level of evidence II, degree of recommendation B). Although pain due to bone metastases has been associated with incidental and predictable pain, our patient-specific data showed that only $50 \%$ of the patients reported incidental onset of pain, and fewer than half $(44.3 \%)$ experienced predictable BTcP episodes. This justifies the preference for ROOs over immediaterelease morphine or oxycodone among the pain experts polled in our study. Additionally, most metastatic patients were receiving rapid-onset fentanyl to treat their breakthrough pain (either alone or in combination with other medications), showing that rapid-onset fentanyl preparations are the treatment of choice for BTcP caused by bone metastasis for the specialists in our study.

It is important to stress that the use of ROOs varied significantly according to specialty, with the overall percentage of sublingual fentanyl prescriptions being higher among PC physicians. The administration of immediate-release morphine and NSAIDs for BTcP also differs between specialties: PC physicians had a greater predilection for immediate-release morphine, whereas PU specialists preferred NSAIDs as their second most common option after fentanyl formulations. These results show that the criteria for prescribing pharmaceutical treatments for $\mathrm{BTcP}$ caused by bone metastases is not homogeneous in clinical practice in Spain.

\section{Conclusions}

Overall, the results of our study on patients suffering from bone $\mathrm{BTcP}$ show that important aspects of the management of these patients, such as functionality, HRQoL, or other psychosocial determinants, are often overlooked in routine clinical practice. Additionally, our findings underline the need for treatment protocols and specific referral criteria for BTcP patients with bone metastasis in Spain.

Our results show that rapid-onset fentanyl preparations are the treatment of choice for BTcP caused by bone metastasis in clinical practice in Spain. However, they also reveal the different prescribing practices among $\mathrm{RO}$, $\mathrm{PC}$, and PU specialists treating $\mathrm{BTcP}$ caused by bone metastases and underscores the need for multidisciplinary BTcP management strategies.

\section{Ethical standards}

This study was approved by the Ethics Committee of the Consorcio Hospitalario Provincial de Castellón (Spain). The study was conducted according to the criteria set by the Declaration of Helsinki and each patient signed an informed consent before their inclusion in the study.

\section{Acknowledgments}

We would like to thank the following researchers for their contribution to the research required for this study: Silvia Rodriguez Villalba, José María Nieto-Guerrero Gómez, Jonathan Saavedra Bejarano, Francisco Villegas Estévez, Angel Plasencia Ezaine, Raúl, Sánchez Posada, Ruth Carmona Vigo, Pablo Maradey, Joan Coma Alemany, Carme, Martínez Arribas, Gabriel Triana Martínez, Ferran Nonell Gregori, Jose de la Cueva Aguilera, Jose Antonio Giron Mombiela, Ana Otero Romero, José Antonio Díaz Ricós, Miriam Nuñez Fernández, Gala Serrano, $M^{\text {a }}$ Dolors Torremorell Balagué, Sara Pedraza Fernández, María Teresa Palomino Jiménez, Fernando López Campos, Nuria Piera, Javier Arranz Durán, Víctor Rivas Jiménez, Jorge Jiménez Calero, Ana Belen Mencias Hurtado, María del Mar Hernández García, Toribio Bernabé Gonzélez Guillermo, Pablo Noguera Sánchez, Ricardo Valdés Arribas, Diego Díaz Rodríguez, Alejandro Morales, Jesús Manuel Blanco Suarez, Martín Arcas Molina, Inés Formoso García, Juan Quirós Rivero, Antonio Cañas Pedrosa, Manuel Robles Romo, Miguel Angel Núñez Viejo, Raquel García Gómez, Jose Agustín González Romero, Alejandro De Luis Lillo, Jose Giménez Viudes, Juan Carlos Jiménez Marcelo, Paloma Moreno Ceano, Nuria Domínguez Serrano, Jose Montesdeoca Santana, María Acuña Mora, José Manuel Trinidad Martín-Arroyo, Jose Carlos Villen, Diego Benitez Pareja, María Sol Moral Serrano, Antonio Gallegos Polonio, Luis Fernández Fornos, Pablo Villacé, Mercedes Molina Robles, Silvia Remesal Blanco, Irene Ortiz González, Meritxell Vidal Borràs, Enrique Domínguez Suarez, Laia Navarri, Cristina Nicolau Martorell, Iñigo Nieto Regueira, Inmaculada Herrador Montiel, Ruben Dario Ramírez Vargas, Jose Quero López, $\mathrm{M}^{\mathrm{a}}$ Luisa Tarrasó Gómez, Marcelino Mosquera Pena, Elena Escobar Sánchez, Jordi Marruecos Querol, Maria Dolores Rodrigo Royo, Ane Escribano, Paula Peleteiro Higuero, Cristian Carrasquer Díaz, Gemma Asín Felipe, Marian Segarra Soria, Montserrat Reche García, Susana Plaza Canteli, Eva María Lozano Martín, María Jose Caballero Aceituno, Rafael Ambrosi Centeno, Jose Manuel González Mesa, María Madariaga Muñoz, Martín García Muñoz, Rosa Izquierdo Aguirre, José Javier Martín Ortega, Carlos Ferrer Albiach, Jesús María Mirasol García, Ana Isabel Luque Blanco, Yolanda Sastre Peris, Sandra Guardado Gonzales, María Ana Estornell Gualde, Joaquín Cartagena Sevilla, Maria Espinosa Calvo, Luz Canovas Martínez, Pedro Pablo Escolar Pérez, $M^{a}$ Dolores López Alarcón, 
Esther González Calvo, Inmaculada Muro Castillo, Javier Valencia Julve, María Aurora Viloria Jiménez, Xelo Lapuebla Ferri, Manuel Enguix Castelo.

The authors thank Lucía Perez at Medical Statistics Consulting Sociedad Limitada (Valencia, Spain) for editorial support in writing of this manuscript. Medical Statistics Consulting Sociedad Limitada was contracted by Kyowa Kirin Farmacéutica, S.L.U.

This study was sponsored by Kyowa Kirin Farmacéutica, S.L.U., Spain. Staff from the Medical Department at the sponsoring company were involved in study design, the interpretation of data results, and in the decision to submit the article for publication.

We declare that the data presented in this paper have been partly presented at the 9th World Congress of the World Institute of Pain (WIP 2018) on May 9-12, 2018, and the Multinational Association of Supportive Care in Cancer and the International Society of Oral Oncology (MASCC/ISOO 2018) Annual Meeting on June 28-30, 2018, as a poster.

\section{Disclosure}

CFA and FVE received fees from Kyowa Kirin Farmacéutica, S.L.U., for the design, execution, and coordination of the study. In addition, the following authors declare potential conflicts of interest for individual activities for the industry outside the submitted work: CFA has received payments for consultancies and lecture fees from Astellas Pharma, Janssen,GP-Pharm, and Kyowa Kirin Farmacéutica, S.L.U.

FVE has received payments for consultancies and lecture fees from Grünenthal, Gebro Pharma, Menarini, Takeda, Mylan, Esteve, and Ferrer. MDLA has received payments for consultancies and lecture fees from Kyowa Kirin Farmacéutica, S.L.U., Takeda, Gebro Pharma, Esteve, Teva, and Grünenthal.

$\mathrm{MM}$ has received lecture fees from Kyowa Kirin Farmacéutica, S.L.U., Grünenthal, Mundipharma, Ferrer, and Medtronic.

AC has received payments for consultancies and lecture fees from Takeda, Gebro Pharma, Esteve, Teva, Grünenthal, and Kyowa Kirin Farmacéutica, S.L.U. JA has received payments for consultancies and lecture fees from Esteve, Abstral and Kyowa Kirin Farmacéutica, S.L. U. JMTMA has received payments for consultancies and lecture fees from Grünenthal, Boston Scientific, Gebro Pharma, Angellini Farmaceutica, Takeda, Kyowa Kirin Farmacéutica, S.L.U. and Ferrer. AJJL and ASY are employed by Kyowa Kirin Farmacéutica, S.L.U. The authors report no other conflicts of interest in this work.

\section{References}

1. Reyes Chiquete D ,C González Ortiz, A Mohar Betancourt, A Meneses García. Epidemiología del dolor por cáncer. Revista De La Sociedad Española Del Dolor. 2011;18:118-134.

2. van Den Beuken-van Everdingen MH, Hochstenbach LM, Joosten EA, Tjan-Heijnen VC, Janssen DJ. Update on prevalence of pain in patients with cancer: systematic review and meta-analysis. $J$ Pain Symptom Manage. 2016;51(6):1070-1090 e1079. doi:10.1016/j. jpainsymman.2015.12.340

3. Porta-Sales J, Garzon Rodriguez C, Julia Torras J, Casals Merchan M. [Cancer-related breakthrough pain]. Med Clin (Barc). 2010;135 (6):280-285. doi:10.1016/j.medcli.2010.02.008

4. Jara C, Del Barco S, Gravalos C, et al. SEOM clinical guideline for treatment of cancer pain (2017). Clin Transl Oncol. 2018;20(1):97107. doi:10.1007/s12094-017-1791-2

5. Coleman RE. Clinical features of metastatic bone disease and risk of skeletal morbidity. Clin Cancer Res. 2006;12(20 Pt 2):6243s-6249s. doi:10.1158/1078-0432.CCR-06-0931

6. Li S, Peng Y, Weinhandl ED, et al. Estimated number of prevalent cases of metastatic bone disease in the US adult population. Clin Epidemiol. 2012;4:87-93. doi:10.2147/CLEP.S28339

7. Mercadante S, Arcuri E. Breakthrough pain in cancer patients: pathophysiology and treatment. Cancer Treat Rev. 1998;24(6):425-432.

8. Mercadante S, Radbruch L, Caraceni A, et al. Episodic (breakthrough) pain: consensus conference of an expert working group of the European Association for Palliative Care. Cancer. 2002;94(3):832-839.

9. Portenoy RK, Payne D, Jacobsen P. Breakthrough pain: characteristics and impact in patients with cancer pain. Pain. 1999;81(1-2):129-134.

10. Marras F, Leali PT. The role of drugs in bone pain. Clin Cases Miner Bone Metab. 2016;13(2):93-96. doi:10.11138/ccmbm/2016.13.2.093

11. Nabal M, Librada S, Redondo MJ, Pigni A, Brunelli C, Caraceni A. The role of paracetamol and nonsteroidal anti-inflammatory drugs in addition to WHO Step III opioids in the control of pain in advanced cancer. A systematic review of the literature. Palliat Med. 2012;26 (4):305-312. doi:10.1177/0269216311428528

12. Wiffen PJ, Derry S, Moore RA, et al. Oral paracetamol (acetaminophen) for cancer pain. The Cochrane Database Syst Rev. 2017;7: CD012637. doi:10.1002/14651858.CD003881.pub4

13. Zeppetella G, Ribeiro MD. Opioids for the management of breakthrough (episodic) pain in cancer patients. The Cochrane Database Syst Rev. 2006;(1):CD004311.

14. Fallon M, Giusti R, Aielli F, et al. Management of cancer pain in adult patients: ESMO clinical practice guidelines. Ann Oncol. 2018;29(Supplement_4):iv166-iv191. doi:10.1093/annonc/mdy152

15. Greco MT, Roberto A, Corli O, et al. Quality of cancer pain management: an update of a systematic review of undertreatment of patients with cancer. J Clin Oncol. 2014;32(36):4149-4154. doi:10.1200/JCO.2014.56.0383

16. Ripamonti CI, Santini D, Maranzano E, Berti M, Roila F, Group EGW. Management of cancer pain: ESMO clinical practice guidelines. Ann Oncol. 2012;23(Suppl 7):vii139-vii154. doi:10.1093/annonc/mds233

17. Apolone G, Corli O, Caraceni A, et al. Pattern and quality of care of cancer pain management. Results from the cancer pain outcome research study group. $\mathrm{Br} J$ Cancer. 2009;100(10):1566-1574. doi:10.1038/sj.bjc. 6605053

18. Margarit C, Julia J, Lopez R, et al. Breakthrough cancer pain - still a challenge. J Pain Res. 2012;5:559-566. doi:10.2147/JPR.S36428

19. Working Group Nientemale DEI, Vellucci R, Fanelli G, et al. What to do, and what not to do, when diagnosing and treating breakthrough cancer pain (BTcP): expert opinion. Drugs. 2016;76(3):315-330. doi:10.1007/s40265-015-0519-2 
20. Schneider G, Voltz R, Gaertner J. Cancer pain management and bone metastases: an update for the clinician. Breast Care. 2012;7(2):113120. doi:10.1159/000338579

21. American Pain F. Breakthrough cancer pain: mending the break in the continuum of care. J Pain Palliat Care Pharmacother. 2011;25 (3):252-264. doi:10.3109/15360288.2011.599920

22. Yang P, Cheville AL, Wampfler JA, et al. Quality of life and symptom burden among long-term lung cancer survivors. J Thorac Oncol. 2012;7(1):64-70. doi:10.1097/JTO.0b013e3182397b3e

23. Scharpf J, Karnell LH, Christensen AJ, Funk GF. The role of pain in head and neck cancer recurrence and survivorship. Arch Otolaryngol Head Neck Surg. 2009;135(8):789-794. doi:10.1001/ archoto.2009.107

24. Walker MS, Miller PJ, Namjoshi M, Houts AC, Stepanski EJ, Schwartzberg LS. Relationship between incidence of fracture and health-related quality-of-life in metastatic breast cancer patients with bone metastases. $J$ Med Econ. 2013;16(1):179-189. doi:10.3111/13696998.2012.737883

25. Davies A, Buchanan A, Zeppetella G, et al. Breakthrough cancer pain: an observational study of 1000 European oncology patients. $J$ Pain Symptom Manage. 2013;46(5):619-628. doi:10.1016/j. jpainsymman.2012.12.009
26. Chow E, Zeng L, Salvo N, Dennis K, Tsao M, Lutz S. Update on the systematic review of palliative radiotherapy trials for bone metastases. Clin Oncol. 2012;24(2):112-124. doi:10.1016/j. clon.2011.11.004

27. Smith HS, Mohsin I. Painful boney metastases. Korean J Pain. 2013;26(3):223-241. doi:10.3344/kjp.2013.26.3.223

28. Di Franco R, Falivene S, Ravo V, et al. Management of painful bone metastases: our experience according to scientific evidence on palliative radiotherapy. Anticancer Res. 2014;34(2):1011-1014.

29. NICE. Palliative care for adults: strong opioids for pain relief; 2012. Available from: www.nice.org.uk/cg140. Accessed July 1, 2018.

30. European Oncology Nursing Society guidelines. Breakthrough cancer pain guidelines; 2013 . Available from: https:/www.cancernurse.eu/ documents/EONSBreakthroughCancerPainGuidelines.pdf. Accessed July 1, 2018.

31. Wengstrom Y, Geerling J, Rustoen T. European oncology nursing society breakthrough cancer pain guidelines. Eur J Oncol Nurs. 2014;18(2):127-131. doi:10.1016/j.ejon.2013.11.009

32. Mercadante S. Non pharmacological interventions and non-fentanyl pharmacological treatments for breakthrough cancer pain: a systematic and critical review. Crit Rev Oncol Hematol. 2018;122:60-63. doi:10.1016/j.critrevonc.2017.12.016
Journal of Pain Research

\section{Publish your work in this journal}

The Journal of Pain Research is an international, peer reviewed, open access, online journal that welcomes laboratory and clinical findings in the fields of pain research and the prevention and management of pain. Original research, reviews, symposium reports, hypothesis formation and commentaries are all considered for publication. The manuscrip management system is completely online and includes a very quick and fair peer-review system, which is all easy to use. Visit http:// www.dovepress.com/testimonials.php to read real quotes from published authors. 